\title{
Endoscopic Management of Acute Cholecystitis Following Metal Stent Placement for Malignant Biliary Strictures: A View from the Inside Looking in
}

\author{
Sean Bhalla and Ryan Law \\ Division of Gastroenterology and Hepatology, University of Michigan, Ann Arbor, MI, USA
}

See "Endoscopic Ultrasonography-Guided Gallbladder Drainage as a Treatment Option for Acute Cholecystitis after Metal Stent Placement in Malignant Biliary Strictures" by Fumisato Kozakai, Yoshihide Kanno, Kei Ito, et al., on page 262-268.

Acute cholecystitis is a known adverse event that occurs in a small number of patients who have undergone placement of a self-expandable metal stent (SEMS) in the common bile duct for the treatment of malignant biliary obstruction. ${ }^{1,2}$ In patients with unresectable pancreaticobiliary malignancies, palliative treatment of acute cholecystitis is necessary; however, cholecystectomy has an unacceptable risk/benefit profile and, presumptively, a higher morbidity and mortality rate in this population. The current standard of care in this scenario is percutaneous transhepatic gallbladder drainage (PTGBD) with aspiration alone or cholecystostomy tube placement. ${ }^{3}$ While this procedure is relatively safe and effective, PTGBD in patients with pancreaticobiliary malignancies often necessitates lifelong percutaneous drainage requiring routine catheter maintenance and exchanges, due to the reported risk of recurrent cholecystitis following catheter removal being $41 \% .{ }^{4}$ It is widely recognized that percutaneous catheters are uncomfortable, are difficult to manage, and can adversely the affect quality of life. ${ }^{5}$ With this in mind, there has been an increase

Received: May 8, 2019 Accepted: May 10, 2019

Correspondence: Ryan Law

Division of Gastroenterology and Hepatology, University of Michigan, 3912 Taubman Center, 1500 E. Medical Center Dr., SPC 5362, Ann Arbor, MI 481095362, USA

Tel: +1-734-615-7914, Fax: +1-734-232-4294, E-mail: drryanlaw@gmail.com ORCID: https://orcid.org/0000-0001-7048-9268

cc This is an Open Access article distributed under the terms of the Creative Commons Attribution Non-Commercial License (http://creativecommons.org/ licenses/by-nc/3.0) which permits unrestricted non-commercial use, distribution, and reproduction in any medium, provided the original work is properly cited. in data on endoscopic ultrasound (EUS)-guided transmural GBD in the literature.

In this issue of Clinical Endoscopy, Kozakai et al. ${ }^{6}$ present the clinical outcomes of patients who underwent EUS-GBD for acute cholecystitis following SEMS placement to relieve malignant biliary obstruction. In this single-center retrospective review, 10 patients who underwent EUS-GBD after failure of PTGBD or percutaneous transhepatic gallbladder aspiration (PTGBA) were identified over an 8-year period. The outcomes of these patients were compared to those of a control cohort of patients who underwent the conventional intervention with PTGBD with drain placement $(n=11)$ or PTGBA $(n=27)$. EUS-GBD was technically and clinically successful in $90 \%$ of patients, with an adverse event rate of $40 \%$. Four patients suffered from bile peritonitis, one of whom required surgical management for necrotizing cholecystitis and needed repair for bile duct injury and cholecystectomy. Following EUSGBD, 38\% of patients had recurrent cholecystitis within one month of the procedure due to stent dislodgement. In comparison, the technical success rate for PTGBA and PTGBD was $100 \%$. The clinical success rate was $91 \%$ in the PTGBD group and 63\% in the PTGBA group. Of the 10 patients who did not demonstrate clinical success in the PTGBA cohort, 8 subsequently underwent EUS-GBD. PTGBD catheters were removed in a majority of patients after recovery of cholecystitis at an average of 26 days, with a lower rate of recurrent cholecystitis at $10 \%$. The median survival period for EUSGBD was 259 days compared to 178 days for PTGBD and 
188 days for PTGBA. This study demonstrated that both fully covered SEMSs or plastic stents can be used successfully for EUS-GBD, given that lumen-apposing metal stents (LAMSs) are not approved for EUS-GBD in most countries.

We commend the authors for demonstrating a high technical success rate of EUS-GBD using SEMSs and plastic stents in a unique patient population that developed acute cholecystitis after SEMS placement for malignant biliary stricture. Kozakai et al. ${ }^{6}$ demonstrate that this procedure is a feasible and less invasive option for chronically ill patients unfit for surgical intervention. Furthermore, it appears to be a successful alternative for patients who have had failed percutaneous interventions or in patients who would prefer to avoid an external drainage tube. This study highlights the potentially high adverse event rate and risk for recurrent cholecystitis that is associated with EUS-GBD. This is congruent with the current literature on adverse events such as recurrent cholecystitis, bleeding, peritonitis, perforation, pain, stent migration, and pneumoperitoneum, with an occurrence rate of $6 \%-50 \%{ }^{7-9}$ In this study, recurrent acute cholecystitis occurred due to stent dislodgment. This suggested that the stent type may affect adverse event rates. Furthermore, the authors utilized double pigtail plastic stents or fully covered biliary SEMSs without anti-migration properties to create a conduit for GBD. While all currently available SEMSs and LAMSs are for off-label use in the gallbladder, SEMSs with anti-migration features made by several manufacturers and LAMSs may minimize the adverse event rate related to EUS-GBD. There is no consensus on stent choice for EUS-GBD; however, two recent meta-analyses have shown that EUS-GBD with LAMSs has a high technical success rate of $93 \%-95 \%$ with a low adverse event rate of $10 \%-18 \%$, suggesting equivalent technical and clinical successes with an improved safety profile. ${ }^{9,10}$ SEMSs or LAMSs, which can be delivered and deployed from an electrocautery-enhanced catheter, may also mitigate risk as this approach limits the procedure time and accessory exchanges. However, a well-designed randomized controlled trial comparing LAMSs and non-lumen-apposing stents has not been performed. Furthermore, the use of pigtail stents within the lumen of a SEMS or LAMS may further limit migration as demonstrated by Takagi et al.

In summary, EUS-GBD should be considered in patients with acute cholecystitis stemming from SEMS placement to relieve malignant biliary obstruction. This study demonstrates near equivalence in technical and clinical successes when comparing EUS-GBD to PTGBD; however, EUS-GBD appeared to confer a survival advantage in this small study. The quality of life and patient preferences were not assessed as part of this study. Published studies have reported diminished quality of life in patients with indwelling percutaneous cathe- ters. We believe that migration/stent dislodgement leading to recurrent cholecystitis, which was a major limitation of EUSGBD in this study, can be overcome by utilizing the equipment and techniques listed above, thereby potentially paving the way for EUS-GBD as the first-line intervention in patients with acute cholecystitis presenting to centers with therapeutic EUS expertise and with similar clinical circumstances.

This study provided additional data to the growing literature regarding the various approaches to managing acute cholecystitis in a variety of clinical situations. We believe that EUS-GBD is a reasonable minimally invasive technique that can be used to avoid percutaneous tube placement for cholecystitis in patients with pancreaticobiliary malignancies. The current trajectory of EUS-GBD is positive and bright; however, unseating percutaneous techniques in the current treatment algorithm require further refinement of techniques and procedural accessories designed specifically for EUSGBD procedures as well as increased exposure for therapeutic endoscopists wishing to perform these procedures. Long-term follow-up data from large prospective clinical trials comparing treatment strategies and technical variations are necessary to fully define the optimal EUS-GBD techniques that will maximize technical and clinical successes while limiting adverse events.

\section{Conflicts of Interest}

Ryan Law is consultant of Olympus America. Other author has no financial conflicts of interest.

\section{Author Contributions}

Writing-original draft: Sean Bhalla

Writing-review\&editing: Ryan Law

\section{REFERENCES}

1. Suk KT, Kim HS, Kim JW, et al. Risk factors for cholecystitis after metal stent placement in malignant biliary obstruction. Gastrointest Endosc 2006;64:522-529.

2. Isayama $H$, Kawabe $T$, Nakai $Y$, et al. Cholecystitis after metallic stent placement in patients with malignant distal biliary obstruction. Clin Gastroenterol Hepatol 2006;4:1148-1153.

3. Baron TH, Grimm IS, Swanstrom LL. Interventional approaches to gallbladder disease. N Engl J Med 2015;373:357-365.

4. McKay A, Abulfaraj M, Lipschitz J. Short- and long-term outcomes following percutaneous cholecystostomy for acute cholecystitis in highrisk patients. Surg Endosc 2012;26:1343-1351.

5. Peñas-Herrero I, de la Serna-Higuera C, Perez-Miranda M. Endoscopic ultrasound-guided gallbladder drainage for the management of acute cholecystitis (with video). J Hepatobiliary Pancreat Sci 2015;22:35-43.

6. Kozakai F, Kanno Y, Ito K, et al. Endoscopic ultrasonography-guided gallbladder drainage as a treatment option for acute cholecystitis after metal stent placement in malignant biliary strictures. Clin Endosc 2019;52:262-268.

7. Takagi W, Ogura T, Sano T, et al. EUS-guided cholecystoduodenostomy 
for acute cholecystitis with an anti-stent migration and anti-food impaction system; a pilot study. Therap Adv Gastroenterol 2016;9:19-25.

8. Walter D, Teoh AY, Itoi T, et al. EUS-guided gall bladder drainage with a lumen-apposing metal stent: a prospective long-term evaluation. Gut 2016;65:6-8.

9. Manta R, Mutignani M, Galloro G, Conigliaro R, Zullo A. Endoscopic ultrasound-guided gallbladder drainage for acute cholecystitis with a lumen-apposing metal stent: a systematic review of case series. Eur J Gastroenterol Hepatol 2018;30:695-698.

10. Kalva NR, Vanar V, Forcione D, Bechtold ML, Puli SR. Efficacy and safety of lumen apposing self-expandable metal stents for EUS guided cholecystostomy: a meta-analysis and systematic review. Can J Gastroenterol Hepatol 2018;2018:7070961. 